record (at $10 \mathrm{~cm}$ intervals) for the Changwu loess section using the ratio of free $\mathrm{Fe}_{2} \mathrm{O}_{3}$ (Fed) (extracted by dithionite-bicarbonate-citrate method) to total $\mathrm{Fe}_{2} \mathrm{O}_{3}$ widely used by pedologists for characterizing the soil weathering intensity. The new proxy is highly consistent with other indicators of the intensity of pedogenesis: (1) the most developed S4, S5-1 and S5-3 have the highest Fed / Fet ratio; (2) for the three sub-units of S5, the ratio is the highest for S5-1 and lowest for S5-2, thus in close agreement with the macro and micromorphological observations; (3) the ratios for the soil units S6, S7 and S8 are similar to those for S2 and S3; and (4) the susceptibility time-series shows a major shift in amplitude at $\sim 600 \mathrm{ka}$ $\mathrm{BP}$ while that for the new proxy is at $\sim 800 \mathrm{ka}$, similar to that defined according to the paleosols. Therefore the Fed/Fet ratio seems to be a better indicator of the strength of the East Asian summer monsoon than do variations in magnetic susceptibility.

We believe that the strong development of the S4, S5-1 and S5-3 soils is not solely a function of a longer period of soil-formation, but of climate conditions. The data match well with the higher $\delta^{13} \mathrm{C}$ values (marine oxygen isotope stages 11, 13 and 15) in the marine record (see Raymonet al., 1990, Earth and Planetary Science Letters, 97:353-368; and Oppo et al., 1990, Palaeoceanography, 5:43-54). These periods also correspond with the periods of greatest Atlantic-Pacific benthic $\delta^{13} \mathrm{C}$ gradients, suggesting a link with the rate of the deep-water formation in the North Atlantic.

Zhengtang Guo, Tungsheng Liu, Lanying Wei, Naiqin Wu, Huoyuan lu and Wenying Jiang Institute of Geology, Chinese Academy of Sciences, P.O. Box 9825, Beijing 100029, China

\title{
Reconstruction of Paleodlimate in the Loess Plateau using Non-Linear Mathematical Methods
}

In reconstructions of Quaternary climate, most researchers have made quantitative estimates of paleoclimate from linear relations between paleoclimatic proxy records and single climatic factors (e.g. temperature or precipitation), and established non-linear equations related to two climatic parameters such as temperature and precipitation by using response surface analysis. In this study, we use a non-linear inversion method to synthesize the data of three different proxy indicators and reconstruct paleoclimate. 63 records of Magnetic susceptibility (MS), 12 of the total $\mathrm{Fe}_{2} \mathrm{O}_{3}$ (Fet) and 28 of the mollusk species (Vallonia cf. pulchella) were taken from modern surface soils of the Loess Plateau and used as proxy indicators of physical, chemical and biological records. For stratigraphical study, we chose the Luochuan loess section, a standard section for Chinese loess. This section was sampled at intervals of $10 \mathrm{~cm}$ from S1 to S0. A total of 120 samples for MS, Fet and mollusks have been studied. The data for annual mean temperature (AMT) and annual mean precipitation (AMP) at the modern surface soil sites were used to set up the multiple regression climatic functions. Fig. 3 shows temperature and precipitation variation in Luochuan over the past $11,000 \mathrm{ka}$ based on the non-linear inversion method. From Fig. 3, we can see that there are some phase differences in the variations of the three proxy records. However, the AMT and AMP satisfy the non-linear relationship between the three climatic proxies and temperature and precipitation within acceptable limits, indicating a mutually consistent solution of the three climatic proxies.

Naiqin Wu, Houyuan lu, Zhengtang Guo Institute of Geology, Chinese Academy of Sciences, Beijing 100029, China

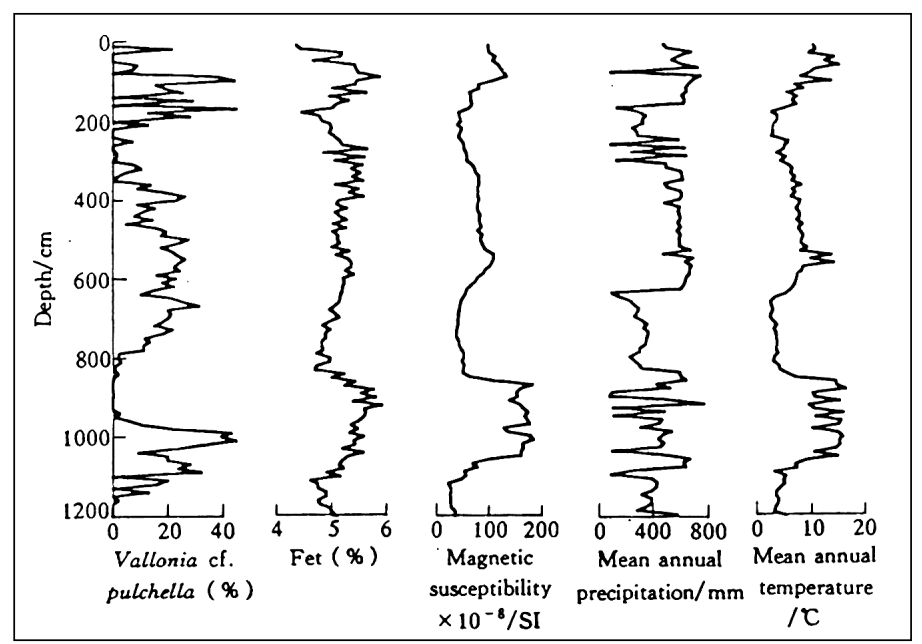

Fig. 3: Variations in the three proxy records of Vallonia $c f$. pulchella, Fet and MS in the Luochuan loess section since the last interglacial together with variations in $A M T$ and $A M P$ as derived from the nonlinear inversion of these three proxy indicators.

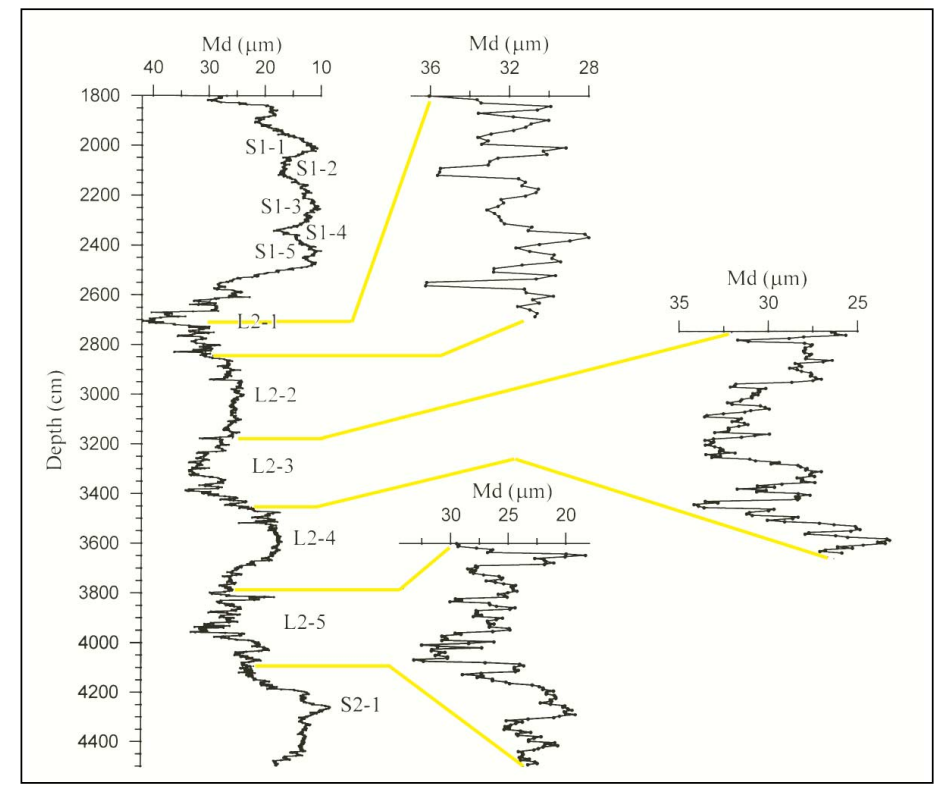

\section{Climatic Instability during the Penultimate Glaciation: Evidence from the Chinese Loess Deposits}

Since the recognition of millennial-scale climatic oscillations in the Greenland ice cores, high-resolution records derived from various deposits all over the world have convincingly demonstrated that paleoclimatic variability of this kind is recorded in different parts of the global climate system, implying that climate instability during the last glacial period may be regarded as a global phenomenon. However, most of the high-resolution proxy records obtained hitherto only cover the last glaciation, and so climatic variability on sub-Milankovitch time scales in the older glacial periods is poorly known. Recently, we generated a high-resolution grain size record at Huining, the northwestern part of the Loess Plateau. The loess-soil sequence accumulated during the last two glacial-interglacial periods is about $45 \mathrm{~m}$ thick in the Huining section. We took samples of this part at $2 \mathrm{~cm}$ intervals. This sample spacing yields an average depositional time resolution of below a hundred years. Examination of the grain size record for the last 